\title{
artigo
}

\section{Absenteísmo da população masculina na assistência à saúde mental: uma revisão narrativa}

\author{
Absentism of the male population in mental health care: a narrative review \\ Ausentismo de la población masculina en la atención de salud mental: una revisión narrativa
}

\begin{abstract}
RESUMO
Objetivo: Compreender os principais motivos que levam os homens a não buscarem auxílio profissional para suas queixas relativas à saúde mental. Método: Revisão de literatura narrativa, adaptada do PRISMA, com busca de artigos nos sítios PubMed, SciELO entre outros, no esquema booleano (man AND mental health) AND (adherence OR services OR risks). Resultado: Encontrou-se de 5.669 artigos, no qual 44 atendiam os critérios de seleção e 15 tinham relação direta com o problema. Via mapa mental, resultados foram agrupados em cinco sessões: aspectos da saúde mental nos homens, hipóteses para baixa procura, imposição de regras sociais, características dos homens que não buscam auxílio e consequências na saúde do homem do não cuidado da saúde mental. Principais causas relatadas para o absenteísmo foram comportamentais - visto que os homens buscam não aparentar fraqueza e vulnerabilidade. Conclusão: Questões sociais e um imperativo comportamental leva os homens a distanciar-se dos serviços de assistência
\end{abstract}

DESCRITORES: Assistência à Saúde Mental; Saúde do Homem; Literatura de Revisão; Absenteísmo.

\section{ABSTRACT}

Objective: To understand the main reasons why men do not seek professional help for their mental health complaints. Method: Review of narrative literature, adapted from PRISMA, with search for articles on PubMed, SciELO and others, in the Boolean scheme (man AND mental health) AND (adherence OR services OR risks). Result: There were 5,669 articles, in which 44 met the selection criteria and 15 were directly related to the problem. Via a mind map, results were grouped into five sessions: aspects of mental health in men, hypotheses for low demand, imposition of social rules, characteristics of men who do not seek help, and consequences for men's health from mental health care. Main causes reported for absenteeism were behavioral - since men try not to show weakness and vulnerability. Conclusion: Social issues and a behavioral imperative leads men to distance themselves from assistance services

DESCRIPTORS: Mental Health Assistance; Men's Health; Review Literature; Absenteeism.

\section{RESUMEN}

Objetivo: Comprender las principales razones por las que los hombres no buscan ayuda profesional para sus problemas de salud mental. Método: Revisión de literatura narrativa, adaptada de PRISMA, con búsqueda de artículos sobre PubMed, SciELO y otros, en el esquema booleano (man AND mental health) AND (adherence OR services OR risks). Resultado: Hubo 5.669 artículos, de los cuales 44 cumplieron con los criterios de selección y 15 estaban directamente relacionados con el problema. A través de un mapa mental, los resultados se agruparon en cinco sesiones: aspectos de la salud mental en los hombres, hipótesis de baja demanda, imposición de reglas sociales, características de los hombres que no buscan ayuda y consecuencias para la salud masculina de la atención en salud mental. Las principales causas reportadas para el ausentismo fueron conductuales, ya que los hombres tratan de no mostrar debilidad y vulnerabilidad. Conclusión: Los problemas sociales y un imperativo conductual llevan a los hombres a distanciarse de los servicios de asistencia.

DESCRIPTORES: Asistencia de salud mental; Salud de los hombres; Revisar la literatura; Absentismo.

RECEBIDO EM: 27/12/2020 APROVADO EM: 07/01/2021

\section{Miline Weis Becker}

Graduandas em Medicina, Universidade da Região de Joinville, UNIVILLE.

ORCID: 0000-0003-1518-7288 
Júlia Carolina Esteves de França

Graduandas em Medicina, Universidade da Região de Joinville, UNIVILLE.

ORCID: 0000-0002-4375-1870

\section{Luana Schlindwein Imhof}

Graduandas em Medicina, Universidade da Região de Joinville, UNIVILLE.

ORCID: 0000-0002-0754-2342

\section{Yasmim Roberta Ferreira}

Graduandas em Medicina, Universidade da Região de Joinville, UNIVILLE.

ORCID: 0000-0003-0241-2925

\section{Luciano Henrique Pinto}

Professor Adjunto, Departamentos de Medicina, Enfermagem e Farmácia, Universidade da Região de Joinville, UNIVILLE, Coordenador do Projeto Integrado ECOSAM.

ORCID: 0000-0003-0250-7502

\section{INTRODUÇÃO}

\section{0} conceito de saúde mental envolve a análise do ser humano como um ser biopsicossocial, onde deve-se levar em consideração todas as relações que ele possui, como por exemplo: relações interpessoais, com o meio ambiente e consigo mesmo. Nesse sentido, as últimas décadas foram marcadas por uma intensa preocupação pela busca do equilíbrio em várias áreas da vida, visando alcançar uma mente saudável ${ }^{1}$. Dentre as questôes levantadas nesse contexto, percebe-se uma preocupação com o impacto do sexo e gênero na saúde mental, uma vez que determinadas patologias psiquiátricas atingem muito mais os homens do que as mulheres, e nota-se um absenteísmo masculino na procura por serviços de ajuda nessa área ${ }^{2}$.

Dessa forma, a relação entre saúde mental e as questões de gênero se apresentam cada vez mais frequentes na atualidade. Assim, tendo em vista os problemas referentes à saúde do homem, torna-se urgente o entendimento do absenteísmo masculino em questões de saúde mental, bem como a procura por ações efetivas que busquem diminuir esses números. $\mathrm{O}$ presente trabalho, trata-se de uma revisão de literatura que busca entender melhor os padrões de atendimento em saúde da população masculina, visando compreender os motivos que levam os homens a não buscarem auxílio profissional para suas queixas - em especial as relacionadas a saúde mental ${ }^{1,2}$.
Assim, tendo em vista os problemas

referentes à saúde

do homem,

torna-se urgente

o entendimento

do absenteísmo

masculino em

questões de saúde

mental, bem como

a procura por ações

efetivas que busquem

diminuir esses

números.
É possível inferir que uma das causas para a carência masculina na procura por ajuda pode estar relacionada com aspectos culturais e ideais masculinos de estigmatização ${ }^{3}$. Segundo Iwamoto et al., existe uma congruência entre as construções relacionadas à masculinidade e os problemas psicológicos entre homens, onde, na tentativa de seguir normas pré-estabelecidas pela sociedade do que é "ser homem", eles colocam-se em risco de desenvolver problemas emocionais, como a depressão ou o abuso de álcool ${ }^{3}$.

Portanto, entende-se que buscar ajuda profissional, para questôes mentais e psicológicas, abrange desde a preocupação com determinado problema até a aceitação do mesmo. Entretanto, muitas pessoas relutam em procurar este tipo de auxílio, por medo de julgamentos, sendo os homens jovens o grupo menos provável de buscá-la ${ }^{4}$. Esse contexto reflete dados levantados pela Organização Mundial da Saúde (OMS), que aponta que quase três vezes mais homens do que mulheres morrem devido ao suicídio no mundo 5 .

Este trabalho por sua vez objetiva entender as causas que levam ao absenteísmo masculino na saúde mental, para levantar reflexões sobre este tema e estratégias para saná-lo.

\section{MÉTOdO}

O processo de pesquisa de revisão narrativa, que consistiu em cinco fases, adap- 
tado do método PRISMA, realizado em julho/agosto de 2020 (Figura 1).

Os descritores eleitos no item na Fase I, do Item A do método e utilizadas no esquema booleano foram: (man AND mental health) AND (adherence OR services OR risks), tendo em vista a questão norteadora da investigação: causas do absenteísmo masculino no cuidado da saúde mental.

Toda atividade foi realizada com avaliação em pares entre pesquisadores e orientador da pesquisa, sendo este o revisor final do trabalho.

\section{RESULTADOS}

Foram encontrados um total de 5.669 artigos, no qual 44 atendiam os critérios de seleção da pesquisa para encontrar proposições que atendesse a dúvida de pesquisa do trabalho; sendo que 15 foram utilizadas neste artigo por ter relação mais próxima com a temática (Figura 2).

Frente a análise dos dados encontrados, via mapa mental, os resultados foram agrupados em cinco sessões a fim de se encon-
Figura 1: Processo de pesquisa bibliográfica utilizado, contendo as fases de busca e seleção, a fim de encontrar artigos que respondam à questão de pesquisa. Adaptado do método PRISMA
I - Estabelecimento dos critérios de inclusảo e exclusăo dos artigos: Eleigăo de ccrno os artigos seriam

buscados, incluido os crikerios para escolha das palavies chaves:

a) Palaveas-chaves presentes do DeCS - Descritcres en ciencias da Saude.

b) Disporibiadade ncs idicmas portugias, ingles e espanhol.

c) Ressça cireta com o objeno de estuso e com a questso ncreadora dele,

d) Publicados nos unimos 10 ancs. Obras taras foram consutadas e consideradas neste trataeho quanoo a selevencia da abordagem histórica foi significatva. movida DOK algume discusseo entre os arligos encontrodos.

e) Näo apresentar corfitos de interesse

II - Pesquisa em sitlos eletrónicos determinados na tase It: Estabelecimento dos critutios ANOIOURNOT para a pesquisa e busca nos sities indexados de maior relevalncia sobre o sema:
ETADA DE BUSCA

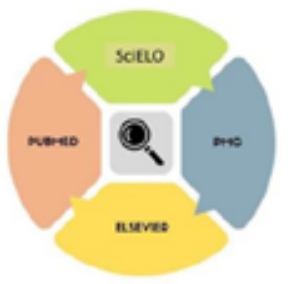

ETADA DE TRIAGEM

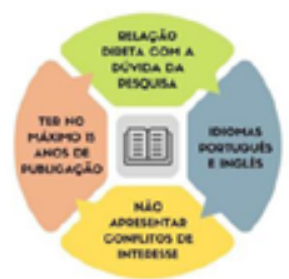

IV - Seleção dos artigos: Os artigos encontrados na listagem apos inclusao das palavras chaves formam eleitcs a partir de très ecapas:

a) Leibura do titulo e avaliaçâo dos mesnos quanto a ligaço com a pergunta da pesculsa.

b) Leitura do Resumo para methor compreens

c) Leitura completa do periodico para seleço em pré-avaliagho quando en dovida da sua contribukplo para a pesoguisa,

V - Coleta đas informaçốes e confrontamento com a đúvida da pesquisa: Uso de mapa mental para abordar o problema da pesquisa a luz das indicaçoes dos autores dos artigos eleitos.

IV - Soleção dos artigos: OE artigos encontrados na listagem ap6s inclusડ̄o das palavras chawes formam eleitios a partir de trés etapas:

a) Leitura do tivio e avaliaçào dos mesmos quanto a ligaça com a pergunta da pesquisa,

b) Leitura do Resumo para molher compresnsto,

c) Leitura completa do poribdico para soloçło om ped-avaliaçlı quando om dúvida da sua contribuiçălo pera e pesquiso. trar a solução para a questão da adesão aos cuidados da saúde mental pelos homens.

As sessões elencadas pelos pesquisadores foram: [1] Aspectos da saúde mental nos homens, [2] hipóteses para baixa procura, [3] a imposição de regras sociais como fator de baixa procura em detrimento de outras causas, [4] características dos homens que não buscam auxílio e [5] consequências na saúde do homem do não cuidado da saúde mental.

\section{DISCUSSÃO}

Os estudos sobre os resultados culminaram nas discussões sobre o tema apresentadas a seguir.

\section{A saúde mental na população mas- culina: uma visão geral}

Entre os grupos que ainda apresentam dificuldades em buscar suporte emocional estão os homens, ao passo que estes carregam um estigma acerca da procura de apoio para os cuidados com a saúde mental, vinculado a crenças negativas para o tratamento ${ }^{6}$. Nesse contexto, estudos indicam que esse absenteísmo masculino quando a busca de serviços de saúde para a saúde mental reflete uma má prática de promoção a saúde dos prestadores, classificando estes serviços como desqualificados ${ }^{7}$. De acordo com Courtenay, os serviços de saúde consideram os homens como invulneráveis e desse modo refletem um declínio na qualidade do atendimento e consequentemente no maior absenteísmo masculino para a demanda de apoio profissional com os cuidados para a saúde mental 8 . Ao passo que entende-se que uma grande parte desses homens procura obter algum método para lidar com a sua saúde mental, e que, de certa forma são infrutíferos ${ }^{9}$.

Através da análise filosófica de Durkheim, em 1897, a respeito do suicídio e as suas diferenças sexuais, outros filósofos partiram dessa ótica e promoveram pesquisas sobre a diferença de buscas de ajuda entre homens e mulheres suicidas. Nesse viés, Möller-Leimkühler trouxe a perspectiva de que a procura externa de ajuda para condições emocionais vai 
Figura 2: Resultados encontrados nos sítios de busca, com os principais critérios adotados para exclusão. Total de 44 artigos foram selecionados e 15 escolhidos para esta pesquisa.

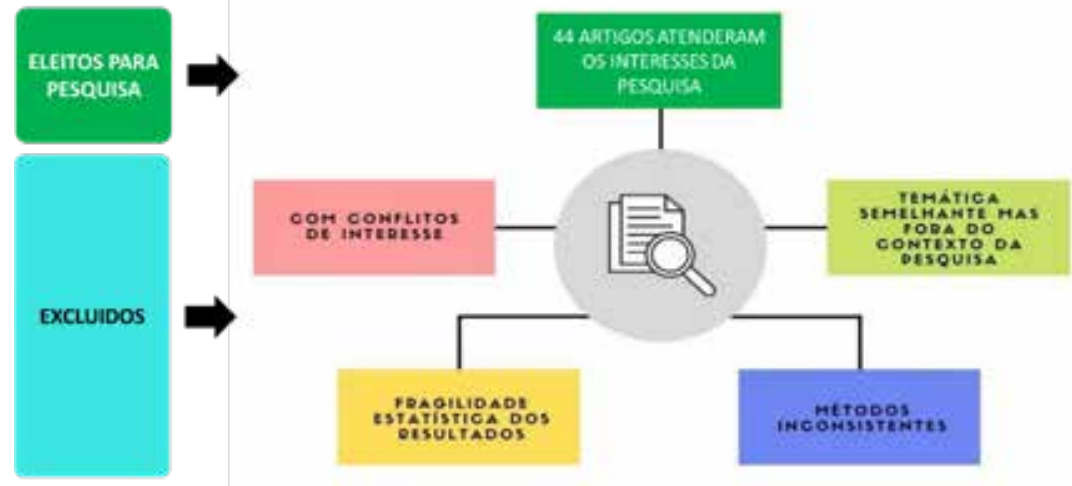

contra os princípios masculinos de autossuficiência. De modo que essa interferência de papéis culturais masculinos dificulta tanto na busca de apoio quanto para o tratamento com a saúde mental dos homens ${ }^{7}$.

Principais hipóteses de causas da baixa procura da população masculina na atenção à saúde mental

Pesquisas indicam que a tendência global de aplicação de normas de gênero leva ao desenvolvimento de uma mentalidade de masculinidade, cujo pilar central é a autossuficiência 9,10. Este aspecto chave leva os homens a acreditarem que, ainda durante os períodos mais difíceis de suas vidas, não devem procurar ajuda - seja ela profissional ou em seu meio de convivência - pois isso seria uma forma de demonstrar fraqueza ${ }^{9,10}$.

Ademais, além de não procurar ajuda, um estudo aponta para possíveis falhas do sistema de saúde e do próprio serviço de saúde - no que diz respeito aos critérios diagnósticos para transtornos de humor depressivos - alegando que os sinais e sintomas característicos não compreendem verdadeiramente os indícios apresentados por homens em quadro de depressão ${ }^{9}$.

Ainda, a falta de abertura para o diálogo sobre o assunto associado a possíveis adversidades na infância, como abusos

\section{De modo}

\section{que essa}

interferência de

papéis culturais

masculinos dificulta

tanto na busca de

apoio quanto para

o tratamento com

\section{a saúde mental}

\section{dos homens.}

emocionais, pode promover a abstenção na busca por cuidados da saúde mental por homens ${ }^{11}$. A literatura mostra que vi- vências abusivas na infância podem refletir em patologias mentais na vida adulta ${ }^{11}$. Soma-se a isso o fato que homens evitam expressar suas emoções por normas e regras sociais, as quais são reproduzidas já nas primeiras fases da vida ${ }^{3}$.

Igualmente, é importante ressaltar o papel das questões familiares referente ao problema'. Segundo Carvalho et al., muitas internações psiquiátricas masculinas estão relacionadas ao abuso de álcool e outras drogas, sendo que umas das causas desta situação é a pressão social sobre o homem como provedor financeiro da família 12. Sendo assim, por pouco expressarem suas apreensões e preocupações, muitos deles procuram nas drogas uma saída para seus conflitos emocionais, o que consequentemente gera problemas psíquicos ${ }^{13}$.

Por fim, outro motivo que pode contribuir para o absenteísmo masculino nos serviços de saúde psicológica e psiquiátrica são os fatores ocupacionais. Embora este fator esteja muito relacionado com contextos sociais e culturais, existem muitas situações estressoras que diferem entre os gêneros dentro das ofertas de trabalho ${ }^{14}$.

\section{Imposição de regras sociais como fator de baixa procura}

São diversas as hipóteses sugeridas pelas literaturas recentes relacionadas ao absenteísmo da população masculina na procura da atenção à saúde mental 9-12,14. Diante disso, percebe-se que a imposição de normas e regras sociais é o fator causal mais enfático do problema da pesquisa, tendo em vista que tal pressão social voltada ao sexo masculino é a causa de diversas outras hipóteses levantadas nas literaturas, como a falta de diálogo, autossuficiência e abuso de álcool e drogas ${ }^{3}$.

Principais características dos indivíduos de sexo masculino que não procuram serviços de saúde

Conforme pesquisas realizadas nos Estados Unidos, não somente aspectos comportamentais são associados a uma baixa procura de serviços de saúde, mas também características como idade, estado civil, IMC e gravidade dos sintomas ${ }^{15}$. Os re- 


\section{artigo}

Becker, M.W.; França, J.C.E.; Imhof, L.S.; Ferreira, Y.R.; Pinto, L.H.;

Absenteísmo da população masculina na assistência à saúde mental: uma revisão narrativa

sultados são indicativos de que pacientes mais velhos; não casados ou em relacionamento estável; com IMC normal; e com sintomas mais graves costumam buscar mais frequentemente ajuda profissional. Enquanto pacientes mais jovens; casados ou em relacionamento estável; em sobrepeso ou obesidade; e com sintomas mais amenos não possuem o hábito de referir aos serviços de atendimento à saúde com frequência desejada ${ }^{15}$.

\section{Principais consequências da menor procuram de ajuda na atenção à saú- de mental}

A baixa procura de apoio para os cuidados com a saúde mental dos homens se relaciona com índices aumentados de suicídios entre o sexo masculino, nesse sentido, um estudo realizado na Austrá- lia relata que, em 2015, mais de $75 \%$ das pessoas que cometeram suicídio eram homens ${ }^{10}$. Isto posto, teorias foram desenvolvidas com a finalidade de justificar a causa dessa diferença de mortes por suicídio entre homens e mulheres, em que se destaca o convencionalismo masculino em não procurar por ajuda para fins de saúde mental ${ }^{10}$.

Outrossim, as consequências dessa falta de busca por ajuda para a saúde mental masculina reflete de diversas formas na qualidade de vida desses homens, de forma que pode resultar na maior incidência de suicídios, no tratamento ineficaz da depressão, no aumento de uso se substâncias que prejudicam tanto a saúde física quanto mental, e outros fatores que interferem no bem estar desses indivíduos. Sendo assim, o não aproveitamento dos serviços de saú- de implica no agravamento da saúde mental masculina?.

\section{CONCLUSÃO}

É notório que há, de fato, uma maior taxa de absenteísmo na procura por assistência a saúde mental por indivíduos do sexo masculino. Tal problema deve-se a uma série de fatores, como pressão social, falta de diálogo sobre o assunto, falha nos critérios diagnósticos e vivências abusivas na infância. Nesse sentido, tais questões desencadeiam na piora da saúde mental, uso de substâncias e maiores taxas de suicídio.

Dessa forma, pretende-se, através desta revisão de literatura, promover o conhecimento acerca da saúde mental na população masculina a fim de melhorar o atendimento à saúde deste grupo populacional.

\section{REFERÊNCIAS}

1. Organização Pan-Americana da Saúde (OPAS). OPAS/OMS Brasil - ONU destaca necessidade urgente de aumentar investimentos em serviços de saúde mental durante a pandemia de COVID-19 [Internet]. 2020 [cited 2020 Nov 9]. Available from: https://www.paho.org/bra/index.php?option=com_content\&view=article\&id=6170:onu-destaca-necessidade-urgente-de-aumentar-investimentos-em-servicos-de-saude-mental-durante-a-pandemia-de-covid-19\&ltemid=839;

2. Affleck W, Carmichael V, Whitley R. Men's Mental Health: Social Determinants and Implications for Services. Canadian Journal of Psychiatry. 2018;

3. Iwamoto DK, Brady J, Kaya A, Park A. Masculinity and Depression: A Longitudinal Investigation of Multidimensional Masculine Norms Among College Men. Am J Mens Health. 2018;

4. Lynch L, Long M, Moorhead A. Young Men, Help-Seeking, and Mental Health Services: Exploring Barriers and Solutions. Am J Mens Health. 2018;

5. Organização Pan-Americana da Saúde (OPAS). OPAS/OMS Brasil - Uma pessoa morre por suicídio a cada 40 segundos, afirma OMS [Internet]. 2019 [cited 2020 Nov 9]. Available from: https:// www.paho.org/bra/index.php?option=com_content\&view=article\&id=6017: suicidio-uma-pessoa-morre-a-cada-40-segundos-afirma-oms\&ltemid=839;

6. Krill Williston S, Roemer L, Vogt DS. Cultural and service factors related to mental health beliefs among post-9/11 veterans. Int J Soc Psychiatry. 2019;65(4):313-21;

7. River J. Diverse and Dynamic Interactions: A Model of Suicidal Men's Help Seeking as It Relates to Health Services. Am J Mens Health. 2018;
8. Courtenay WH. Constructions of masculinity and their influence on men's well-being: A theory of gender and health. Soc Sci Med. 2000;

9. Bilsker D, Fogarty AS, Wakefield MA. Critical Issues in Men's Mental Health. Canadian Journal of Psychiatry. 2018;

10. King KE, Schlichthorst M, Spittal MJ, Phelps A, Pirkis J. Can a documentary increase help-seeking intentions in men? A randomised controlled trial. J Epidemiol Community Health. 2018;

11. Ports KA, Lee RD, Raiford J, Spikes P, Manago C, Wheeler DP. Adverse Childhood Experiences and Health and Wellness Outcomes among Black Men Who Have Sex with Men. J Urban Heal. 2017;

12. Carvalho KL, Terra MG, Moreschi C, Siqueira DF de, Mello A de L, Gamermann A. Characteristics of Mental Health Hospitalizations in Rio Grande Do Sul General Hospitals. Reme Rev Min Enferm. 2019;23:1-8;

13. Lam LCW, Wong CSM, Wang MJ, Chan WC, Chen EYH, Ng RMK, et al. Prevalence, psychosocial correlates and service utilization of depressive and anxiety disorders in Hong Kong: the Hong Kong Mental Morbidity Survey (HKMMS). Soc Psychiatry Psychiatr Epidemiol. 2015;

14. Milner A, Scovelle AJ, King TL, Marck CH, McAllister A, Kavanagh $A M$, et al. Gendered working environments as a determinant of mental health inequalities: A protocol for a systematic review. Int J Environ Res Public Health. 2019;

15. Parent MC, Hammer JH, Bradstreet TC, Schwartz EN, Jobe T. Men's Mental Health Help-Seeking Behaviors: An Intersectional Analysis. Am J Mens Health. 2018; 